\title{
Diagnóstico y tratamiento de los tumores malignos de piel
}

\author{
José Eduardo Telich Tarriba, ${ }^{1}$ Andrea Monter Plata, ${ }^{2}$ \\ André Víctor Baldín, ${ }^{1}$ Armando Apellaniz Campo ${ }^{1}$
}

\section{Resumen}

El cáncer de piel se ha convertido en un problema de salud pública debido a su alta incidencia y al porcentaje considerable que ocupa dentro de las principales neoplasias malignas. Existen diversos factores de riesgo para desarrollar esta patología, y se observó que la exposición a los rayos UV se encuentra dentro de los más importantes. Para su estudio se divide en dos tipos: el melanoma y cáncer de piel no melanoma, que a su vez se clasifica en carcinoma basocelular y espinocelular. La mayoría de los casos son prevenibles y tienen buena respuesta al tratamiento cuando son diagnosticados oportunamente; por ello, debe considerarse tema de relevancia dentro de la práctica médica.

Palabras clave: Cáncer de piel, melanoma, carcinoma espinocelular, carcinoma basocelular.

\section{INTRODUCCIÓN}

El cáncer de piel es la neoplasia maligna más común a nivel mundial y su incidencia ha ido al alza en las últimas décadas. En Estados Unidos se registran anualmente más de dos millones de casos de cáncer de piel, ${ }^{1,2}$ mientras que en México el Registro Histopatológico de Neoplasias

\footnotetext{
${ }^{1}$ Departamento de Cirugía Plástica, Estética y Reconstructiva, Hospital General "Dr. Manuel Gea González", Ciudad de México, México.

2 Departamento de Cirugía Plástica, Instituto Nacional de Ciencias Médicas y Nutrición "Salvador Zubirán", Ciudad de México, México.
}

Correspondencia:

Dr. Armando Apellaniz-Campo

Correo electrónico: dr.apellaniz@gmail.com

Aceptado: 24-11-2016.

Este artículo puede ser consultado en versión completa en http:// www.medigraphic.com/actamedica

\section{Summary}

Skin cancer has become a public health problem due to its high incidence and the considerable percentage that it occupies within the main malignant neoplasias. There are several risk factors to develop this pathology, and it was observed that exposure to UV rays is among the most important. For its study, it is divided into two types: melanoma and non-melanoma skin cancer, which in turn is classified into basal cell and squamous cell carcinoma. Most cases are preventable and respond well to treatment when they are diagnosed promptly; it is, therefore, to be considered a relevant topic within the medical practice.

Key words: Skin cancer, melanoma, basal-cell carcinoma, squamous-cell carcinoma.

Malignas reportó que el $14.6 \%$ de las neoplasias malignas a nivel nacional corresponden a cáncer de piel. ${ }^{3}$

El cáncer de piel se divide en dos grandes grupos: melanoma y no melanoma; dentro de este último se engloban el carcinoma basocelular y el espinocelular, así como tumores poco comunes como linfomas cutáneos, tumores malignos de anexos o de células de Merkel. ${ }^{4,5}$

Método: Se utilizó la base de datos PubMed y se realizó una búsqueda con las palabras clave: non melanoma skin cancer, basal cell carcinoma y squamous cell carcinoma; asimismo, se activó el filtro publication dates: 5 years y se revisaron las referencias de los artículos seleccionados.

\section{CÁNCER DE PIEL NO MELANOMA}

El carcinoma basocelular (CBC) y el carcinoma espinocelular (CEC) son los tumores malignos más comunes a nivel mundial; el primero es el responsable de un $80 \%$ de los casos. ${ }^{6}$

Ambas neoplasias comparten factores de riesgo para su desarrollo; dentro de los principales se encuentran la exposición a radiación ultravioleta, los fototipos I y II (piel 
clara, cabello rubio o rojo, ojos claros), edad avanzada, exposición crónica a arsénico o a radiación ionizante, inmunosupresión y algunas alteraciones genéticas como mutaciones en el gen p53 o xeroderma pigmentoso. ${ }^{7}$

La etiología del cáncer de piel es multifactorial; se observa como principal agente etiológico la radiación UVB, la cual induce mutaciones a nivel del ADN, afectando directamente la vía Hedgehog, lo que activa la replicación de células pluripotenciales en la epidermis. Asimismo, la mutación de otros genes como RAS y el gen supresor de tumores p53 asume un papel importante en la patogenia. ${ }^{8,9}$

\section{CARCINOMA BASOCELULAR}

El CBC es el cáncer de piel más común: se calcula que anualmente hay 2.8 millones de casos nuevos en EUA; de ellos, un 95\% de los casos ocurren entre los 40 y 79 años de edad; afecta más a hombres, con una relación 1.5:1 con respecto a las mujeres.

Se trata de una neoplasia con bajo potencial metastásico y crecimiento lento, pero localmente invasiva y destructiva; deriva de las células basales de la epidermis y sus apéndices. ${ }^{4}$ Afecta la región de cabeza y cuello aproximadamente en un $70 \%$ de los casos; de ellos, el $26 \%$ se localiza en la nariz. Está presente en la región del tronco en un 15\%; en manos y genitales ocurre de manera esporádica. ${ }^{10}$

Existen distintas variantes clínicas de presentación del $\mathrm{CBC}$, entre las que se encuentran las siguientes:

- Nodular: es la forma más común, abarca hasta un $60 \%$ de los casos; generalmente se presenta como una lesión solitaria, rosada, perlada, con telangiectasias y de bordes definidos. Si se ulcera se considera nóduloulcerativo.

- Superficial: representa aproximadamente 30\% de los casos; se observa como una placa eritematosa poco indurada, predomina en el tronco y puede confundirse con tiñas o eccema.

- Morfeiforme o esclerosante: constituye el 5\% de los casos; se caracteriza por ser una lesión aplanada o ligeramente elevada, de coloración blanco-amarillenta o rosada e indurada, similar a una cicatriz. Presenta alta incidencia de márgenes positivos tras la escisión y se considera el subtipo más agresivo.

- Otras: en menor porcentaje se encuentran las formas micronodular, infiltrativa y basoescamosa; se consideran variantes agresivas de CBC con alta tasa de recurrencia. ${ }^{11}$

No existe una lesión precursora universal; sin embargo, el nevo de Jadassohn puede presentar en el $15 \%$ de los casos un CBC en su superficie. El CBC tiende a invadir tejidos profundos a través de vías de menor resistencia como periostio, pericondrio, fascia o la placa tarsal. La invasión a hueso, cartílago y músculo es poco usual, y menos del $0.5 \%$ de los casos presentan metástasis a ganglios linfáticos, pulmón o hueso. Estos se relacionan con lesiones profundas y variantes agresivas como el basoescamoso y tienen supervivencia menor a 10 meses. $^{12}$

El CBC puede tratarse de diversas formas dependiendo de tres factores: la variante clínica, su localización y el tamaño. Las prioridades del abordaje deben enfocarse en alcanzar la erradicación completa del tumor y conseguir resultados funcionales óptimos y estéticos aceptables. ${ }^{13}$

Dentro de las opciones de tratamiento se encuentra el manejo quirúrgico. La resección quirúrgica convencional es una de las técnicas más utilizadas; se ha visto que en lesiones bien circunscritas menores a dos centímetros de diámetro, una escisión con márgenes clínicos de cuatro milímetros resulta en una eliminación completa en más del $95 \%$ de los casos. Tiene las ventajas de poder ser realizada en forma rápida, ser relativamente económica y obtener información histopatológica de control. Sus principales desventajas son ser un procedimiento invasivo que sacrifica cantidades variables de tejido sano. ${ }^{14}$

La cirugía de Mohs es la técnica quirúrgica preferida para $\mathrm{CBC}$ de alto riesgo. Se trata de un procedimiento en el que se realizan cortes delgados del tejido en forma secuencial, los cuales son analizados inmediatamente

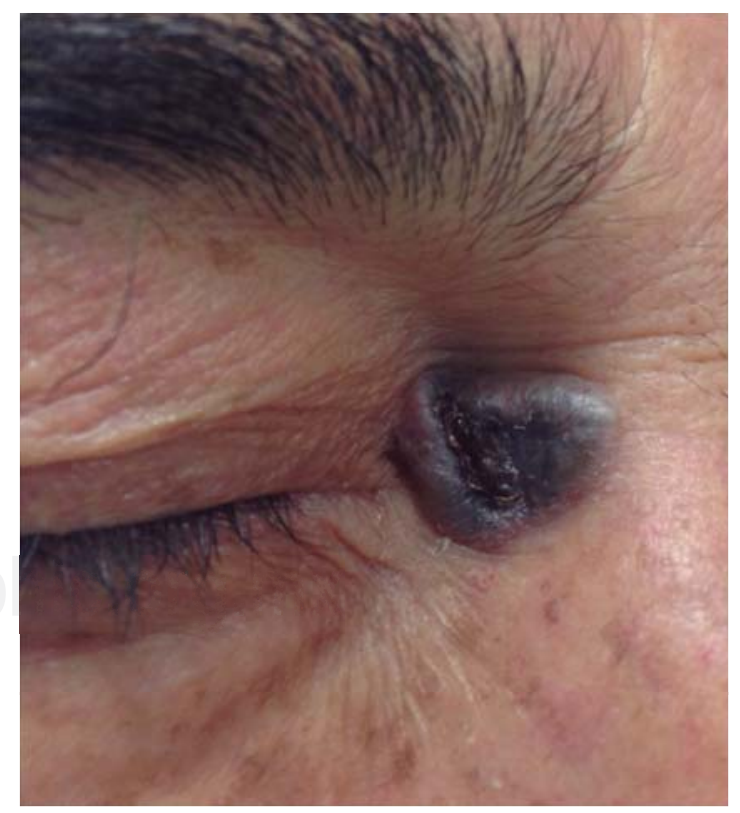

Figura 1. Carcinoma basocelular nodular con zona de ulceración central. 
al microscopio hasta obtener márgenes libres de tumor; ofrece rangos de curación hasta en 99\%, con significativa preservación de tejido. Está indicada principalmente en tumores localizados en áreas cosméticamente sensibles (periorbitaria, periauricular o paranasal), variantes agresivas (morfeiforme o esclerosante), tumores recurrentes, márgenes pobremente definidos en tejido cicatricial y en casos de invasión perineural. Sus principales desventajas son la duración y el costo del procedimiento. ${ }^{15}$

Existen varias alternativas para el manejo farmacológico tópico; dentro de ellas se encuentra el imiquimod, que es un modulador de la respuesta inmunológica, indicado también en formas superficiales. Se aplica una formulación al $5 \%$ cinco veces a la semana durante seis semanas, con una tasa de curación de hasta $95 \%$.

El 5-FU interfiere en la síntesis de ADN; está indicado sólo en estirpes superficiales en zonas no críticas. Se aplica una formulación al $5 \%$ dos veces al día por seis semanas, con una tasa de curación del $80 \%{ }^{16}$

La terapia fotodinámica es un tratamiento que utiliza porfirina fotosensibilizante sobre el sitio a tratar, el agente es absorbido por las células cancerosas mientras la luz produce que el agente fotosensibilizador reaccione con el oxígeno, lo que produce destrucción celular y de los vasos nutricios, con la consiguiente activación del sistema inmune. Algunos estudios reportan que esta terapia puede ser igualmente efectiva que la cirugía o radioterapia, con las ventajas de ser un procedimiento ambulatorio, menos invasivo y más preciso. Sin embargo, no existen resultados a largo plazo. ${ }^{17}$

La radioterapia está indicada en el tratamiento adyuvante en tumores recurrentes, tumores irresecables y pacientes de edad avanzada. ${ }^{18}$

El pronóstico de los individuos con $\mathrm{CBC}$ es excelente; sin embargo, si no son tratados a tiempo, pueden presentar gran morbilidad debido a la destrucción local de tejidos. Los enfermos requieren evaluaciones periódicas: durante el primer año se realizan cada tres meses, el segundo cada seis y posteriormente de forma anual hasta los cinco años. El 80\% de las recurrencias aparecen en los siguientes cinco años. ${ }^{19}$

\section{CARCINOMA ESPINOCELULAR}

El CEC es la segunda neoplasia maligna de piel más común: comprende aproximadamente $20 \%$ de los casos; sin embargo, tiene una tasa de mortalidad mayor que el CBC. Deriva de la proliferación de los queratinocitos de la epidermis y sus anexos, con capacidad de producir metástasis a ganglios regionales u otros órganos. Tiene un crecimiento rápido y aparece con frecuencia sobre lesiones precancerosas. ${ }^{20}$

Comparte factores de riesgo con el CBC; sin embargo, existen factores de riesgo relacionados específicamente con el desarrollo de CEC, entre los que se encuentran zo- nas de inflamación crónica en la piel (quemaduras, lupus discoide, fístulas y osteomielitis), infecciones virales (VPH) y humo de tabaco. ${ }^{21}$

La queratosis actínica es la principal lesión precursora. Se caracteriza por presentar máculas eritematosas con escama, histológicamente similares al CEC. Se ha observado que 5\% de las lesiones progresan a CEC; sin embargo, aproximadamente el $65 \%$ de los CEC derivan de este tipo de lesión.

La enfermedad de Bowen se considera un CEC in situ; se caracteriza por una lesión escamosa sobre una placa eritematosa de crecimiento lento. ${ }^{22}$

Clínicamente, el CEC aparece con mayor frecuencia en la cara, manos y antebrazos; representa alrededor del $60 \%$ de los tumores de la oreja. Se caracteriza por una pápula o placa rosa, frecuentemente escamosa y en ocasiones ulcerada. ${ }^{23}$

El CEC se subdivide en distintos tipos dependiendo de sus características clínicas; entre ellos encontramos los siguientes:

- Verrucoso: se presenta como lesiones exofíticas y de crecimiento lento, común en palmas y plantas, tiene riesgo bajo de metástasis.

- Ulcerativo: es de crecimiento rápido y localmente invasivo, presenta bordes elevados con ulceración central, se caracteriza por tener comportamiento agresivo y suele metastatizar a ganglios linfáticos.

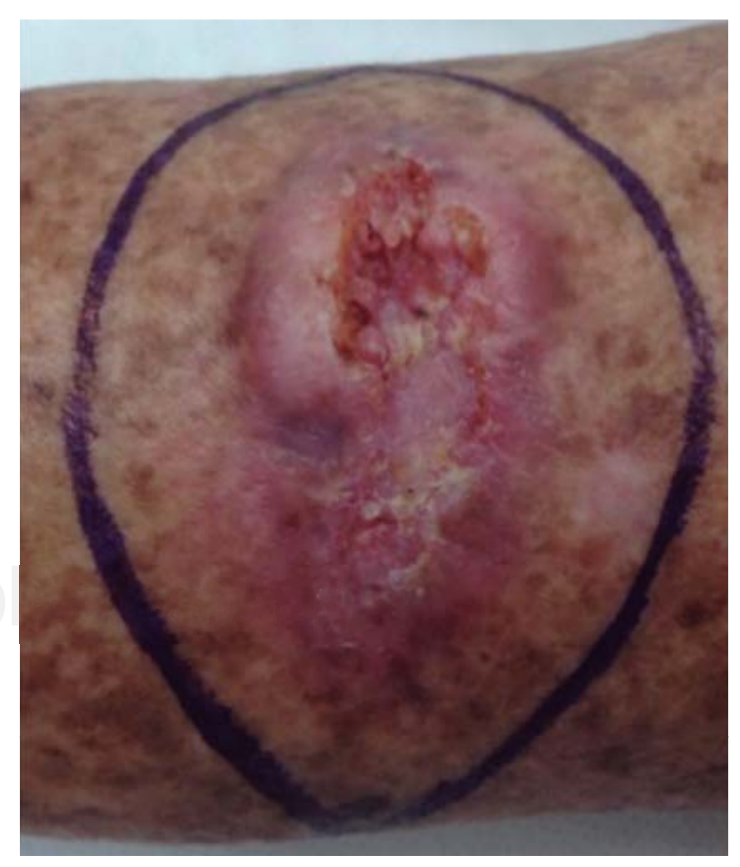

Figura 2. Carcinoma espinocelular ulcerativo. 
- Úlcera de Marjolin: proviene de lesiones con inflamación crónica (quemaduras, fístulas, osteomielitis), tiene un periodo de latencia de 32.5 años en promedio y frecuentemente metastatiza a ganglios linfáticos.

- Subungueal: se observa como cambios escamosos en el lecho ungueal, edema eritematoso y dolor localizado seguido de un nódulo que se ulcera. ${ }^{24}$

EI CEC puede tratarse de diversas formas dependiendo del riesgo de recurrencia locorregional y metástasis, factores de importancia a determinar al momento de elegir la terapéutica a emplear.

Para el tratamiento se utiliza la resección quirúrgica convencional; para lesiones menores de dos centímetros, en regiones de bajo riesgo o con extensión hasta dermis, se sugieren márgenes de cuatro milímetros; para lesiones mayores de dos centímetros, regiones de alto riesgo o con extensión hasta celular subcutáneo, se requieren márgenes de seis milímetros. La cirugía de Mohs otorga una tasa de curación de hasta 95\% y recurrencia a cinco años de $3.1 \%$, en comparación con $8 \%$ en la resección tradicional. ${ }^{25}$

El manejo farmacológico tópico está indicado exclusivamente en queratosis actínicas o enfermedad de Bowen. La crioterapia y el uso de electrofulguración o curetaje están indicados en lesiones pequeñas, enfermedad de Bowen y aquellas limitadas a regiones estéticamente sensibles como labios, párpados y oídos. ${ }^{26}$

En todos los casos es necesario determinar la extensión de la enfermedad hacia ganglios linfáticos. En aquéllos con ganglios palpables o biopsia por aguja fina positiva deberá llevarse a cabo linfadenectomía regional con radioterapia adyuvante debido a que aproximadamente un $5 \%$ de los pacientes con CEC desarrollan metástasis, principalmente a ganglios linfáticos regionales. ${ }^{27}$

Los individuos con CEC primarios resecados tienen una supervivencia a cinco años superior al 90\%; alrededor de $75 \%$ de las recurrencias se presentan en los primeros dos años y el $95 \%$ en los primeros cinco. La supervivencia a cinco años disminuye drásticamente al $25 \%$ en los sujetos con enfermedad metastásica. ${ }^{28}$

Se consideran indicadores de alta recurrencia la presencia de lesiones con bordes poco definidos, tumores recurrentes, crecimiento rápido, invasión perineural, poca diferenciación celular e inmunosupresión. ${ }^{29}$ Asimismo, el grosor del tumor se ha asociado con recurrencia local y presencia de metástasis; por ello, en 2010 el American Joint Committee on Cancer (AJCC) publicó la revisión de la guía TNM para la etapificación del CEC, en la cual la clasificación T dentro de este sistema considera el tamaño del tumor y otras características de alto riesgo, como el grosor del tumor (> $2 \mathrm{~mm}$ y nivel de Clark $>$ IV), la localización anatómica, invasión perineural (PNI) y la diferenciación histológica (mal o no diferenciado), lo que representa un apoyo en la valoración del pronóstico (Cuadro 1). ${ }^{30}$

\section{MELANOMA}

$$
\text { Epidemiología }
$$

El melanoma es la quinta forma de cáncer más común en EUA y la décima en México; en nuestro país su epidemiología difiere a las de otras naciones, pues afecta más a mujeres y se presenta principalmente en extremidades inferiores (acral), seguido por tumores en cabeza y cuello, extremidades superiores y tronco.

Se trata de la neoplasia maligna con mayor aumento en incidencia a nivel mundial, con un incremento del $2 \%$ anual. A pesar de causar el 3\% del total de las neoplasias malignas de la piel, es responsable del 75\% de las muertes relacionadas con ellas; su alta letalidad se relaciona con que suele diagnosticarse en etapas avanzadas y tiene una baja respuesta a tratamientos sistémicos. ${ }^{31}$

Es una neoplasia maligna derivada de melanocitos, células originarias de la cresta neural que se localizan en la unión dermoepidérmica.

El riesgo global para el desarrollo de melanoma es del $2 \%$. Algunos factores de riesgo para su desarrollo son la edad mayor a 50 años, historial familiar de melanoma, presencia de nevos atípicos, múltiples nevos (> 25) y enfermedades asociadas a mutaciones genéticas de $p 16, B R A F$ o MC1R. La exposición solar es el principal contribuyente en la aparición de melanoma debido al efecto deletéreo de los rayos UV en la síntesis y reparación de ADN, especialmente en individuos de piel clara. ${ }^{32}$

Cuadro 1. Clasificación AJCC del tumor primario (T) para el carcinoma espinocelular.

\section{Estadio}

Tx Primario que no puede ser evaluado

TO Sin evidencia de tumor primario

Tis Carcinoma in situ

T1 Tumor $<2 \mathrm{~cm}$ en su mayor dimensión con menos de dos factores de alto riesgo

T2 Tumor $>2 \mathrm{~cm}$ de diámetro o tumor de cualquier tamaño con más de dos factores de alto riesgo

T3 Tumor con invasión de maxila, mandíbula, órbita o hueso temporal

T4 Tumor con invasión de esqueleto o invasión perineural de la base del cráneo 
Se han descrito múltiples lesiones precursoras para melanoma, entre las que se incluyen las siguientes:

- Nevo congénito gigante: se trata de una lesión mayor a 20 centímetros de longitud con un riesgo de malignización del cinco al 20\%, por lo que se recomienda la resección profiláctica.

- Nevos atípicos o displásicos: son mayores que los nevos adquiridos, de cinco a 12 milímetros aproximadamente.

- Melanoma in situ/léntigo maligno: lesión precursora con presencia de células atípicas que no penetra la lámina basal y pueden derivar de un nevo displásico.

- Nevo de Spitz: lesión benigna - también conocida como melanoma juvenil- encontrada en niños y adultos jóvenes. Se caracteriza por ser bien delimitada y elevada; no es una lesión premaligna, pero es difícil distinguirla del melanoma. ${ }^{33}$

Los melanomas pueden aparecer de novo o a partir de una lesión precursora. Durante la exploración física de los pacientes se debe identificar la presencia de nevos atípicos, facilitado por el ABCDE del melanoma:

- A-simetría.

- B-ordes irregulares.

- C-olor no homogéneo.

- D-iámetro mayor a seis milímetros.

- E-volución.

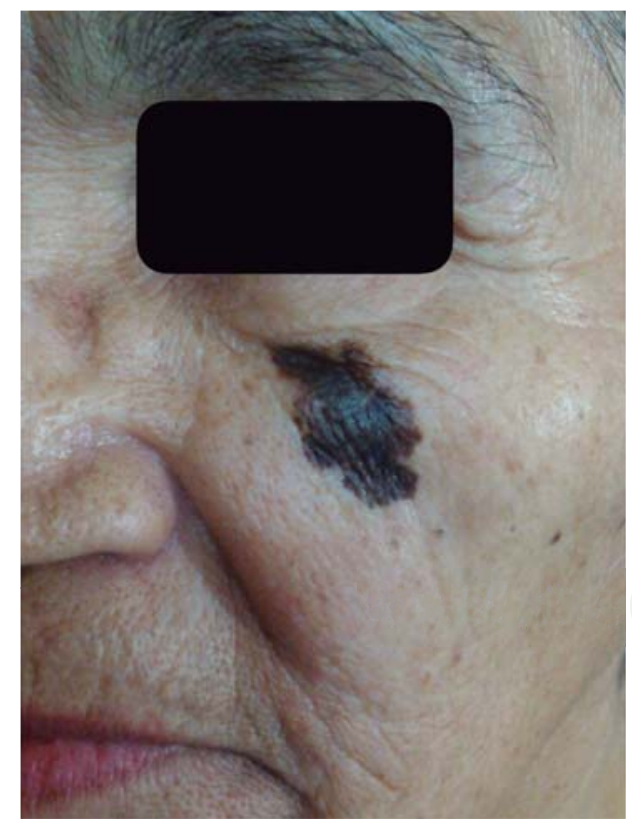

Figura 3. Melanoma en la región facial. Nótese asimetría de la lesión y mala definición de los bordes.
Hasta el $60 \%$ de los melanomas pueden ser diagnosticados exclusivamente por las características clínicas, lo que puede aumentar hasta un $80 \%$ al emplear fotografía de cuerpo completo, microscopia láser o imagen multiespectral. $^{34}$

Dentro de las variantes clínicas del melanoma podemos encontrar los siguientes tipos:

- Diseminado superficial: es el más común, representa alrededor del $70 \%$ de los casos. Se produce de nevos preexistentes, afecta principalmente la región del tronco en hombres y las piernas en mujeres. Presenta una fase de crecimiento horizontal que puede ir desde seis meses hasta seis años antes del crecimiento vertical.

- Nodular: abarca del 15 al 30\% de los casos; es agresivo, típicamente surge de novo, afecta principalmente a hombres y se caracteriza por presentar únicamente una fase de crecimiento vertical.

- Melanoma léntigo maligno: constituye el 10\% de los casos, es el tipo menos agresivo. Claramente está relacionado con exposición solar; deriva del léntigo maligno/mancha de Hutchinson, que tiene crecimiento radial; la transición al crecimiento vertical marca la transición a melanoma.

- Acral lentiginoso: abarca del dos al 8\% de los casos en personas blancas y del 35 al $60 \%$ en aquéllas de color, es el más común en México y se presenta usualmente en palmas, plantas y la región subungueal. Frecuentemente son planos, de tres centímetros, con bordes irregulares y discromía.

- Melanoma amelánico: es una variedad de cualquiera de los anteriores, pero no presenta pigmento y usualmente se diagnostica durante la fase de crecimiento vertical.

- Melanoma mucoso u ocular: comprende el 5\% de los casos y son difíciles de diagnosticar. ${ }^{32}$

El diagnóstico definitivo se basa en la toma de biopsias, las cuales pueden ser escisionales (lesiones $<1.5$ centímetros con margen de dos milímetros) o incisionales ( $>1.5$ centímetros o en zonas de riesgo estético); deben evitarse las biopsias por rasurado debido a que no permiten valorar la profundidad de la lesión. ${ }^{15}$

Los resultados histopatológicos permitirán clasificar la enfermedad dependiendo de su profundidad de acuerdo con los niveles de Breslow. El índice de Clark únicamente se emplea en tumores menores a un milímetro, dependiendo del nivel anatómico de la invasión:

- $\mathrm{I}=$ Epidermis.

- $\quad I I=$ Dermis papilar.

- $\quad$ III = Unión dermis papilar/reticular. 
- $\quad \mathrm{IV}=$ Dermis reticular.

- $\quad V=$ Tejido subcutáneo.

La etapificación de la enfermedad se basa en el sistema TNM (Cuadro 2). Los métodos diagnósticos complementarios (TAC, PET) deben solicitarse con base en la etapa clínica inicial del individuo para definir la extensión de la enfermedad. ${ }^{35}$

El tratamiento dependerá de la extensión de la enfermedad, ya sea local, regional o sistémica.

En caso de ser local, la resección amplia de la lesión primaria es el pilar del manejo del sujeto con melanoma, lo que permite el control de la enfermedad. El tamaño de los márgenes dependerá de la profundidad de la lesión (Cuadro 3). ${ }^{36}$

En casos de biopsia previa, se ampliarán los márgenes midiendo a partir del límite de la cicatriz. Durante la resección no se debe incluir la fascia debido a que aumenta el riesgo de diseminación y no mejora la supervivencia.

En la enfermedad regional, el involucro de nódulos linfáticos es uno de los principales factores determinantes de supervivencia en las personas con melanoma.

La biopsia de ganglio centinela se basa en la teoría de que las metástasis ocurren inicialmente en el primer nódulo linfático de una región. Permite etapificar correctamente la enfermedad en el 97\% de los casos; sin embargo, no mejora la supervivencia. Se indica en melanomas con más de un milímetro de profundidad. El procedimiento

\begin{tabular}{cccccc}
\multicolumn{3}{c}{ Cuadro 2. Etapificación AJCC para melanoma. } \\
\hline \multicolumn{2}{c}{ Tumor } & \multicolumn{2}{c}{ Nódulos } & \multicolumn{2}{c}{ Metástasis } \\
\hline T1 & $<1.0 \mathrm{~mm}$ & $\mathrm{~N} 1$ & 1 & M0 & Sin \\
T2 & $1.01-2.0 \mathrm{~mm}$ & $\mathrm{~N} 2$ & 203 & $\mathrm{M} 1$ & Con \\
T3 & $\begin{array}{c}2.01-4.0 \mathrm{~mm} \\
\text { T4 }\end{array}$ & $\mathrm{N} 3$ & $>4$ & & \\
T4 & $>4.01 \mathrm{~mm}$ & & & & \\
\hline
\end{tabular}

\begin{tabular}{|c|c|}
\hline Espesor del tumor & Márgenes clínicos recomendados \\
\hline In situ & $0.5-1.0 \mathrm{~cm}$ \\
\hline$<1.0 \mathrm{~mm}$ & $1.0 \mathrm{~cm}$ \\
\hline $1.2 \mathrm{~mm}$ & $1-2 \mathrm{~cm}$ \\
\hline $2.01-4 \mathrm{~mm}$ & $2 \mathrm{~cm}$ \\
\hline$>4 \mathrm{~mm}$ & $2 \mathrm{~cm}$ \\
\hline
\end{tabular}

se lleva a cabo con la inyección local de tecnecio-99 y un colorante sobre la lesión junto con la escisión local amplia; en pacientes con biopsia positiva o ganglios palpables, se realiza disección de ganglios linfáticos. ${ }^{37}$

En individuos con etapa clínica IV el pronóstico es sombrío debido a la falta de terapias adyuvantes capaces de controlar la enfermedad. La media de supervivencia es de 10 meses. Entre los fármacos indicados se encuentra el cisplatino y la dacarbazina. Existe investigación activa sobre el uso de anticuerpos monoclonales, vacunas y citocinas.

Se define como recurrencia local a la reaparición del tumor a menos de dos centímetros de la lesión original en un periodo de tres a cinco años debido a una resección incompleta del tumor primario. La resección quirúrgica con márgenes estrechos es el tratamiento de elección ya que no se ha observado mejoría en la supervivencia con escisiones amplias.

La metástasis en tránsito es la presencia de un tumor en piel o tejido subcutáneo a dos o más centímetros del melanoma primario y antes del primer relevo linfático regional. Las personas que las presentan deben ser estudiadas para excluir la presencia de enfermedad sistémica. De no existir evidencia de enfermedad diseminada, los tumores deben ser resecados con márgenes libres, mientras que en los casos con diseminación se deberá complementar el control local con terapia sistémica.

Los principales factores pronósticos de la enfermedad son la profundidad de la lesión, la presencia de ulceración y el índice mitótico. ${ }^{38}$

\section{CONCLUSIONES}

El cáncer de piel es la neoplasia más frecuente en el ser humano y su incidencia continúa en aumento. La exposición a los rayos UV representa el principal factor para el desarrollo de cualquiera de sus tipos.

En la mayoría de las ocasiones la afección es local; no obstante, en nuestro medio generalmente los sujetos son diagnosticados de forma tardía, lo que conlleva mayores retos para el manejo y tratamiento.

Como médicos requerimos conocer las características de cada una de las lesiones para poder referir al paciente con el especialista de manera oportuna y realizar el diagnóstico exacto, determinar el tratamiento apropiado e intervenir adecuadamente en el progreso de la enfermedad.

\section{REFERENCIAS}

1. Lomas A, Leonardi-Bee J, Bath-Hextall F. A systematic review of worldwide incidence of nonmelanoma skin cancer. Br J Dermatol. 2012; 166 (5): 1069-1080. 
2. Mohan SV, Chang AL. Advanced basal cell carcinoma: epidemiology and therapeutic innovations. Curr Dermatol Rep. 2014; 3: 40-45.

3. SINAIS/SINAVE/DGE/SALUD/Perfil epidemiológico de los tumores malignos en México. México: 2011; 45.

4. Gandhi SA, Kampp J. Skin cancer epidemiology, detection and management. Med Clin North Am. 2015; 99 (6): 1323-1335.

5. Reinstadler DR, Sinha UK. Uncommon cutaneous neoplasms of the head and neck. Facial Plast Surg Clin North Am. 2012; 20 (4): 483491.

6. Xiang F, Lucas R, Hales S, Neale R. Incidence of non-melanoma skin cancer in relation to ambient UV radiation in white populations, 1978-2012 empirical relationships. JAMA Dermatol. 2014; 150 (10): 1063-1071.

7. Ouyang YH. Skin cancer of the head and neck. Semin Plast Surg. 2010; 24 (2): 117-126.

8. Madan V, Lear JT, Szeimies RM. Non-melanoma skin cancer. Lancet. 2010; 375 (9715): 673-685.

9. Gordon R. Skin cancer: an overview of epidemiology and risk factors. Semin Oncol Nurs. 2013; 29 (3): 160-169.

10. Wu PA. Epidemiology and clinical features of basal cell carcinoma. UpToDate. 2014.

11. Firnhaber JM. Diagnosis and treatment of basal cell and squemous cell carcinoma. Am Fam Physician. 2012; 86 (2): 161-168.

12. Ibrahim O, Gastman B, Zhang A. Advances in diagnosis and treatment of nonmelanoma skin cancer. Ann Plast Surg. 2014; 73 (5): 615-619.

13. Ermertcan AT, Hellings PW, Cingi C. Nonmelanoma skin cancer of the head and neck: nonsurgical treatment. Facial Plast Surg Clin North Am. 2012; 20 (4): 445-454.

14. Akcam TM, Gubisch W, Unlu H. Nonmelanoma skin cancer of the head and neck: surgical treatment. Facial Plast Surg Clin North Am. 2012; 20 (4): 455-471.

15. NCCN Clinical Practice Guidelines in Oncology. Basal cell skin cancer, Version 2.2016. Disponible en: https://www.nccn.org/professionals/ physician_gls/f_guidelines.asp

16. Metterle L, Nelson C, Patel N. Intralesional 5-fluorouracil (FU) as a treatment for nonmelanoma skin cancer (NMSC): A review. J Am Acad Dermatol. 2016; 74 (3): 552-557.

17. Bahner JD, Bordeaux JS. Non-melanoma skin cancers: photodynamic therapy, cryotherapy, 5-fluorouracil, iquimod, diclofenac, or what? Facts and controversies. Clin Dermatol. 2013; 31 (6): 792-798.

18. Rong Y, Zuo L, Shang L, Bazan JG. Radiotherapy treatment for nonmelanoma skin cancer. Expert Rev Anticancer Ther. 2015; 15 (7): 765-776.

19. Song F, Qureshi AA, Giovannucci EL, Fuchs CS, Chen WY, Stampfer $\mathrm{MJ}$ et al. Risk of a second primary cancer after non-melanoma skin cancer in white men and women: a prospective cohort study. PLoS Med. 2013; 10 (4): e1001433.

20. Veierød MB, Couto E, Lund E, Adami HO, Weiderpass E. Host characteristics, sun exposure, indoor tanning and risk of squamous cell carcinoma of the skin. Int J Cancer. 2014; 135 (2): 413-422.
21. Leonardi-Bee J, Ellison T, Bath-Hextall F. Smoking and the risk of nonmelanoma skin cancer: systematic review and meta-analysis. Arch Dermatol. 2012; 148 (8): 939-946.

22. Prieto-Granada C, Rodriguez-Waitkus P. Cutaneous squamous cell carcinoma and related entities: Epidemiology, clinical and histological features, and basic science overview. Curr Probl Cancer. 2015; 39 (4): 206-215.

23. Martorell-Calatayud A, Sanmartín-Jiménez O, Cruz-Mojarrieta J, Guillén-Barona C. Cutaneous squamous cell carcinoma: defining the high-risk variant. Actas Dermosifiliogr. 2013; 104 (5): 367-379.

24. Kallini J, Hamed N, Khachemoune A. Squamous cell carcinoma of the skin: epidemiology, classification, management, and novel trends. Int J Dermatol. 2015; 54 (2): 130-140.

25. Divine J, Stefaniwksy L, Reddy R, Padilla P, Hagele T, Patel NS et al. A comprehensive guide to the surgical management of nonmelanoma skin cancer. Curr Probl Cancer. 2015; 39 (4): 216-225.

26. Metterle L, Russell JS, Patel NS. An overview of the medical management of nonmelanoma skin cancer. Curr Probl Cancer. 2015; 39 (4): 226-226.

27. NCCN Clinical Practice Guidelines in Oncology. Squamous cell skin cancer, Version 1.2016. Disponible en: https://www.nccn.org/ professionals/physician_gls/f_guidelines.asp

28. Lansbury L, Bath-Hextall F, Perkins W, Stanton W, Leonardi-Bee J. Interventions for non-metastatic squamous cell carcinoma of the skin: systematic review and pooled analysis of observational studies. BMJ. 2013; 347: f6153.

29. Kyrgidis A, Tzellos TG, Kechagias N, Patrikidou A, Xirou P, Kitikidou $K$ et al. Cutaneous squamous cell carcinoma (SCC) of the head and neck: risk factors of overall and recurrence-free survival. Eur J Cancer. 2010; 46 (9): 1563-1572.

30. Broughman ND, Tan ST. The incidence and risk factors of metastasis for cutaneous squamous cell carcinoma - Implications on the T-classification system. J Surg Oncol. 2014; 110 (7): 876-882.

31. Siegel R, Ma J, Zou Z, Jemal A. Cancer statistics, 2014. CA Cancer J Clin. 2014; 64 (1): 9-29.

32. Eggermont AM, Spatz A, Robert C. Cutaneous melanoma. Lancet. 2014; 383 (9919): 816-827.

33. Olsen CM, Carroll HJ, Whiteman DC. Estimating the attributable fraction for cancer: a meta-analysis of nevi and melanoma. Cancer Prev Res (Phila). 2010; 3 (2): 233-245.

34. Dickson PV, Gershenwald JE. Staging and prognosis of cutaneous melanoma. Surg Oncol Clin N Am. 2011; 20 (1): 1-17.

35. Ott PA, Berman RS. Surgical approach to primary cutaneous melanoma. Surg Oncol Clin N Am. 2011; 20 (1): 39-56.

36. NCCN Clinical Practice Guidelines in Oncology. Melanoma, Version 3.2016. Disponible en: https://www.nccn.org/professionals/ physician_gls/f_guidelines.asp

37. Farma JM, Kulkarno N, Hsu C. Surgical management of primary and recurrent melanoma. Surg Oncol Clin N Am. 2015; 24 (2): 239-247.

38. Johnson TM. Guidelines of care for the management of primary cutaneous melanoma. J Am Acad Dermatol. 2013; 69 (6): 1049-1050. 